\title{
InP/Silicon Hybrid External-Cavity Lasers (ECL) Using Photonic Wirebonds as Coupling Elements
}

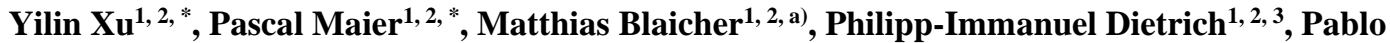 \\ Marin-Palomo ${ }^{1}$, Wladislaw Hartmann ${ }^{1, \text { b) }}$, Muhammad Rodlin Billah ${ }^{1,2,3}$, Ute Troppenz ${ }^{4}$, \\ Martin Möhrle ${ }^{4}$, Sebastian Randel ${ }^{1}$, Wolfgang Freude ${ }^{1}$, Christian Koos ${ }^{1,2,3}$ \\ ${ }^{1}$ Institute of Photonics and Quantum Electronics (IPQ), Karlsruhe Institute of Technology (KIT), Engesserstr. 5, 76131 Karlsruhe, Germany \\ ${ }^{2}$ Institute of Microstructure Technology (IMT), KIT, Hermann-von-Helmholtz-Platz 1, 76344 Eggenstein-Leopoldshafen, Germany \\ ${ }^{3}$ Vanguard Automation GmbH, Gablonzerstr. 10, 76185 Karlsruhe, Germany \\ ${ }^{4}$ Fraunhofer Heinrich-Hertz-Institute (HHI), Einsteinufer 37, 10587 Berlin, Germany \\ a) Now with: Nanoscribe GmbH, Hermann-von-Helmholtz-Platz 1, 76344 Eggenstein-Leopoldshafen, Germany \\ b) Now with: Institute of Physics, University of Munster, Wilhelm-Klemm-Str. 10, 48149 Münster, Germany \\ $\left.{ }^{*}\right) Y$. Xu and P. Maier contributed equally to this work \\ yilin.xu@kit.edu,pascal.maier@kit.edu, christian.koos@kit.edu
}

\begin{abstract}
We demonstrate an InP/Silicon integrated ECL using a photonic wirebond as intra-cavity coupling element. In our proof-of-concept experiments, we demonstrate $50 \mathrm{~nm}$ tuning range, SMSR above $40 \mathrm{~dB}$, and linewidths of $750 \mathrm{kHz}$.
\end{abstract}

OCIS codes: $130.3120,130.5460,140.5960,220.4000$

\section{Introduction}

Tunable lasers are key building blocks of integrated optics. In this context, external-cavity lasers (ECL) are particularly interesting, opening the possibility to combine optical gain in III-V materials with narrowband optical feedback offered by advanced photonic integrated circuits. Such devices can, e.g., be realized by wafer-bonding InP gain material onto pre-processed silicon photonics $(\mathrm{SiP})$ circuits, thereby paving a path towards highly scalable monolithic integration [1]. However, the underlying processes are comparatively complex, requiring ultra-clean and extremely smooth surfaces as well as precise control over environmental conditions and materials. As a consequence, this approach is mainly suited for high-volume applications that justify the associated technological overhead. Alternatively, optical feedback and gain can be realized on separate dies, e.g., by connecting an InP-based reflective semiconductor optical amplifier (RSOA) to a silicon photonic [2] or a silicon nitride [3] external feedback circuit. This concept allows the components to be optimized and tested individually, and it facilitates thermal decoupling of the gain element from the temperature sensitive frequency feedback. On the other hand, high-precision alignment of the dies with respect to each other is required to achieve efficient optical coupling. Scalability to high volumes is therefore limited.

In this paper, we demonstrate a new class of hybrid external-cavity lasers (ECL) that exploits 3D-printed photonic wirebonds [4] for connecting InP RSOA to SiP external-cavity circuits. In this approach, the shape of the 3D-printed photonic wirebond can be adapted to the positions and the mode-field sizes of the chips at either end, thereby avoiding high-precision alignment and replacing additional spot size converters [5]. In first proof-of-concept experiments, we demonstrate tuning ranges of more than $50 \mathrm{~nm}$, SMSR above $40 \mathrm{~dB}$, and linewidths of $750 \mathrm{kHz}$. While leaving room for further improvements, these results clearly demonstrate the potential of the approach.

\section{Device Concept}

The concept of a hybrid external-cavity laser (ECL) with an intra-cavity photonic wirebond is shown in Fig.1(a). The device consists of two dies - an InP RSOA and a silicon photonic external-cavity circuit, the latter comprising a $2.2 \mathrm{~mm}$ long silicon waveguide (WG) including a spiral-shaped phase-tunable section and a frequency-selective feedback structure. The $600 \mu \mathrm{m}$ long C-band RSOA has a back facet with a high-reflectivity (HR) coating (90\% reflectivity against air) and an angled front facet $\left(9^{\circ}\right)$ with anti-reflection (AR) coating against polymer $(n=1.56)$. At a bias current of $100 \mathrm{~mA}$ the small-signal gain is $23 \mathrm{~dB}$, and the saturation output power is $12 \mathrm{dBm}$. A photonic wirebond connects the RSOA to a down-tapered (taper tip width $180 \mathrm{~nm}$ ) silicon strip waveguide with $500 \mathrm{~nm}$ width and $220 \mathrm{~nm}$ height. Passing the $350 \mu \mathrm{m}$ long cavity phase tuner, the external resonator is completed with the tunable feedback structure. Frequency selectivity is provided by two Vernier ring resonators in add-drop configuration with diameters $62 \mu \mathrm{m}(\mathrm{R} 1)$ and $67 \mu \mathrm{m}(\mathrm{R} 2)$, resulting in slightly different free spectral ranges. From transmission measurements (grating coupler GC 1 to GC 3; see Fig.1(a)) we find Q-factors of about 25000 for the ring resonators. The phase shifter tunes the Fabry-Perot resonances of the external resonator to the maximum mirror reflectivities. Rings and phase shifter are tuned by heating. The silicon chip has been fabricated in a standard CMOS line using $193 \mathrm{~nm}$ deepUV lithography. 

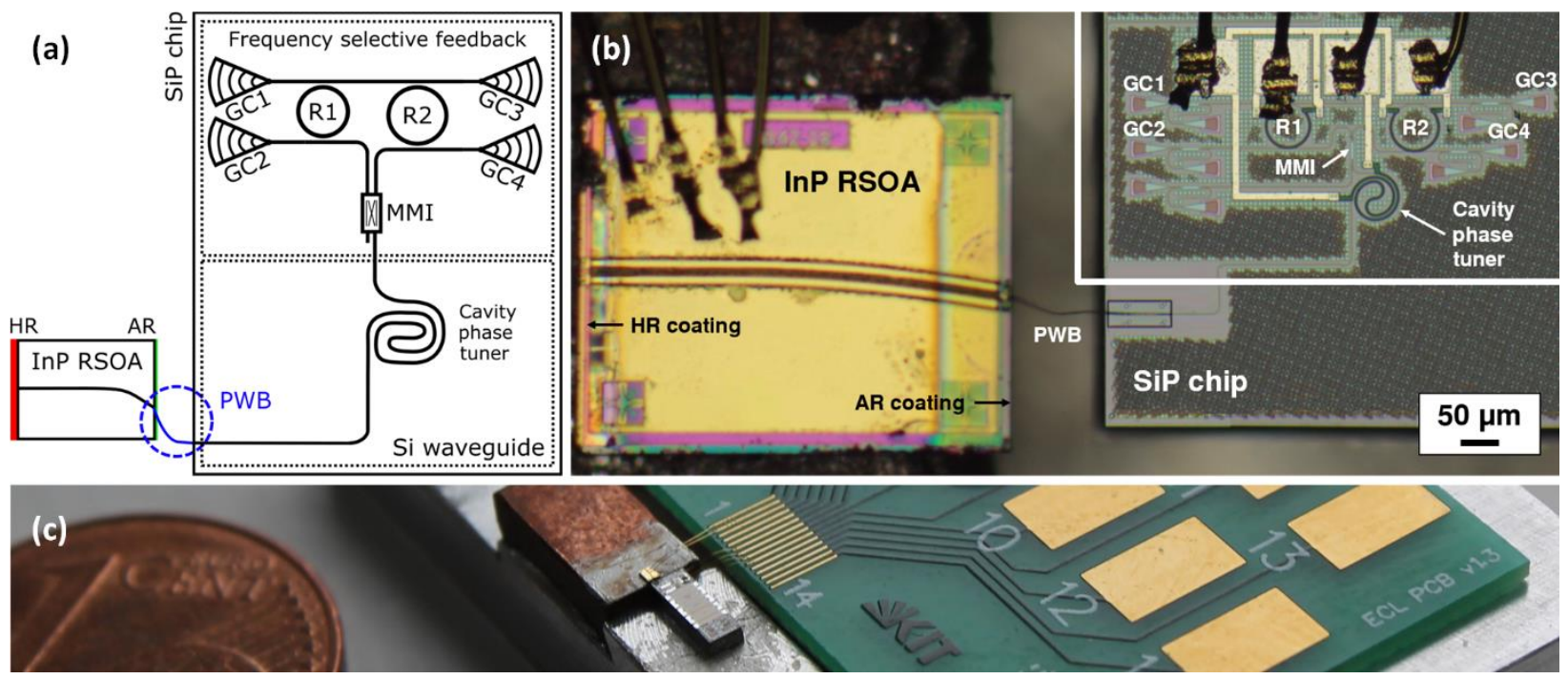

Fig. 1: Concept of a hybrid ECL with intra-cavity photonic wirebond. The device consists of an InP RSOA and a SiP external-cavity circuit, the latter comprising a $2.2 \mathrm{~mm}$ long silicon waveguide (WG) including a spiral-shaped phase-tunable section and a frequency-selective feedback structure. The $600 \mu \mathrm{m}$ long RSOA has a back facet with a HR coating (90\% reflectivity against air) and an angled front facet ( $9^{\circ}$ ) with AR coating against polymer $(n=1.56)$. A photonic wirebond connects the RSOA to a down-tapered (taper tip width $180 \mathrm{~nm}$ ) silicon strip waveguide on the $\mathrm{SiP}$ external-cavity. Passing the $350 \mu \mathrm{m}$ long cavity phase tuner, the external resonator is completed with the tunable feedback structure. Two symmetrically coupled (coupling gap $180 \mathrm{~nm}$ ) Vernier ring resonators in add-drop configuration with diameters $62 \mu \mathrm{m}(\mathrm{R} 1)$ and $67 \mu \mathrm{m}(\mathrm{R} 2)$ provide frequency selectivity. (a) Schematic depicting the building blocks of the device. (b) Microscope image of the assembled device with the previously described building blocks indicated. (c) View of the ECL module alongside electrical assembly.

\section{Module Assembly}

In a first step, the RSOA is glued to a copper heat sink with thermally conductive silver-filled glue (H20E, EPOTEK $\left.{ }^{\circledR}\right)$. The copper heat sink and the silicon chip are then coarsely aligned to each other and glued to a common aluminium submount (as seen in Fig.1(c)). The PWB trajectory is aware of the chip positions and of the oblique emission from the angled RSOA facet. The polymer PWB comprises a taper (widest cross-section $4 \mu \mathrm{m} \times 2.4 \mu \mathrm{m}$ ) for matching the mode field diameter on the InP side to the actual polymer waveguide of the bond (cross-section $2.4 \mu \mathrm{m} \times 1.6 \mu \mathrm{m}$ ). A polymer-to-silicon double-taper transition [6] connects the polymer waveguide to the silicon strip. An additional hold structure is added on the RSOA facet for mechanical stability.

Fabrication is done in-situ by a two-photon lithography step in a negative-tone photoresist (Vanguard Automation $\mathrm{GmbH}, n=1.53$ ). Fabrication is fully automated. The proper writing position is found by rastering and imaging the object with the focused writing beam at low intensity before starting the actual two-photon polymerization process. In the vicinity of the RSOA edge a position-dependent dose correction compensates for the shadowing. The write time amounts to 2:40 min. The fabricated structure is developed in propylene-glycol-methyl-etheracetate (PGMEA), flushed with isopropanol and subsequently blow-dried. Fig.1(b) shows a microscope image of the fully assembled ECL module. We estimate a PWB loss of $3 \mathrm{~dB}$ from amplified spontaneous emission (ASE) measurements before and after module assembly. To this end, the output GC 4 (see Fig.1(a)) is used and the signal levels are compared at a wavelength where the ring resonator $\mathrm{R} 2$ is non-resonant.

\section{Device Performance}

The ECL wavelength is selected by aligning the two ring resonators for a common resonance and by optimizing the cavity phase for a maximum output power, measured at GC 1 . Once the appropriate data are found, they can be stored in a look-up table for later use and for rapid tuning.

From the PI curve in Fig.2(a) we find a threshold current of $30 \mathrm{~mA}$ and a slope efficiency of $35 \mathrm{~mW} / \mathrm{A}$ at a lasing wavelength of $1550 \mathrm{~nm}$. The on-chip power depicted refers to the power in the bus waveguide between R2 and GC 1 . Since our output coupler has four equally strong outputs (GC $1-4)$, we could extract $9 \mathrm{dBm}$ in theory. With a new design which concentrates the optical output power to a single port we can come close to this value. Fig.2(b) depicts the superimposed lasing spectra in steps of $5 \mathrm{~nm}$ within the available tuning range of $1515 \mathrm{~nm}$ to $1565 \mathrm{~nm}$, thereby covering the complete optical C-band. The RSOA bias current has been altered for each operating point to maintain an equal power level. We verify longitudinal single-mode operation by observing the full spectrum across the RSOA gain bandwidth. Mode-hop free operation is observed with a SMSR exceeding $40 \mathrm{~dB}$. 
To find the linewidth of the device we employ the heterodyne method where the output of the ECL is superimposed with a local oscillator in a $90^{\circ}$ optical hybrid which is part of a coherent receiver [7]. The measurements of the ECL linewidth are carried out at a tuned wavelength of $1550 \mathrm{~nm}$ as seen in Fig.2(b). An EDFA is used for amplification of the signal to an appropriate power level. A real-time oscilloscope with $84 \mathrm{GHz}$ electrical bandwidth (Keysight) is used for detection of the beat signal. The local oscillator laser with a linewidth of $10 \mathrm{kHz}$ is tuned to a nearby wavelength to ensure that the intermediate frequency falls into the bandwidth of the coherent receiver. The result from the FM-noise spectrum calculation is shown in Fig.2(c). The instantaneous linewidth is extracted considering the high frequency limit to avoid other noise terms. The result of this fit leads to approximately $S_{F}(f)=2.4 \times 10^{5} \mathrm{~Hz}$ and consequently to a linewidth of $\delta f \approx 750 \mathrm{kHz}$ (blue line).
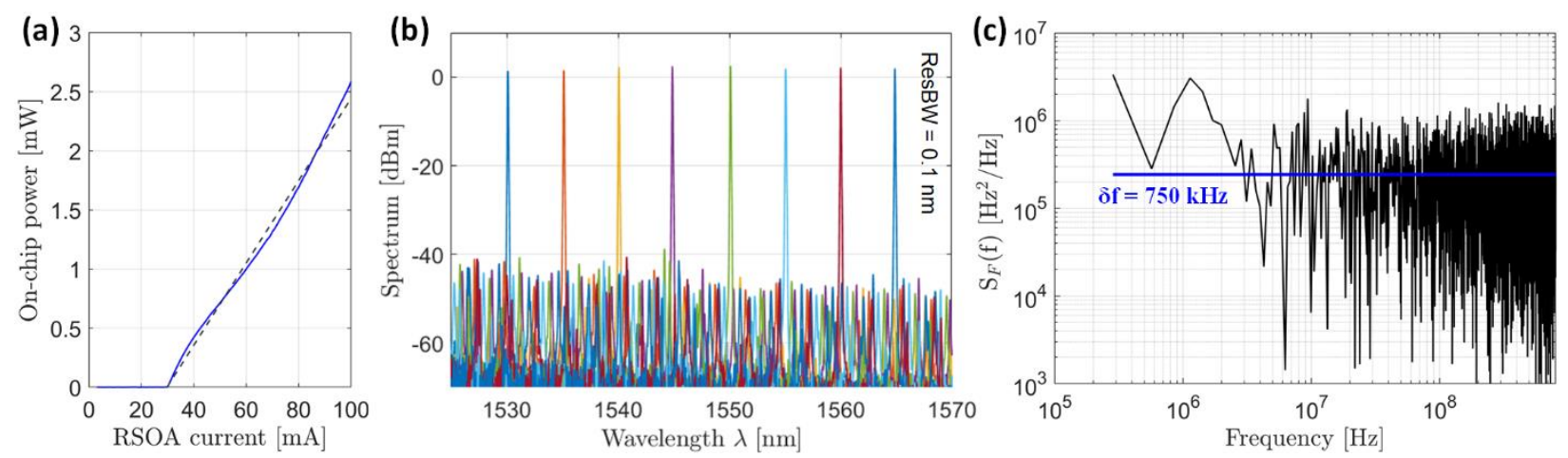

Fig. 2: Device characterization results. (a) PI curve recorded for tuned lasing operation at $1550 \mathrm{~nm}$. A threshold current of $30 \mathrm{~mA}$ and slope efficiency of roughly $35 \mathrm{~mW} / \mathrm{A}$ is found from the measurement. (b) Superimposed lasing spectra recorded in steps of $5 \mathrm{~nm}$ within the available tuning range of $1515 \mathrm{~nm}$ to $1565 \mathrm{~nm}$, thereby covering the complete optical C-band as depicted. The RSOA bias current has been altered for each operating point to maintain an equal power level of $3 \mathrm{dBm}$. We verify longitudinal single-mode operation by observing the full spectrum across the RSOA gain bandwidth. Mode-hop free operation is observed with a SMSR exceeding $40 \mathrm{~dB}$. (c) FM-noise spectrum of ECL module using the heterodyne method and an offline digital coherent receiver. Sampling rate and number of recorded points are $256 \mathrm{GSa} / \mathrm{s}$ and $9 \times 10^{5}$, respectively. The spectrum is first smoothed and subsequently fitted towards high frequencies (blue line) leading to an instantaneous linewidth of $750 \mathrm{kHz}$.

\section{Conclusion}

We demonstrate our assembly approach for a hybrid integrated ECL by connecting an InP gain chip with a Si chip, which provides a tunable frequency-selective feedback by means of a 3D-printed PWB. Our approach does not require high precision alignment of the dies with respect to each other. In a proof-of-principle experiment, we estimate a PWB loss of $3 \mathrm{~dB}$. A tuning range of $50 \mathrm{~nm}(1515 \mathrm{~nm}-1565 \mathrm{~nm})$, an output power of $3 \mathrm{dBm}$, an SMSR above $40 \mathrm{~dB}$ and a linewidth of $750 \mathrm{kHz}$ are found, hence proving the feasibility of our hybrid integration concept. These results compare reasonably well to the performance parameters achieved by other approaches [8], and still leave room for further improvement. As an example, higher $Q$-factors should permit linewidths of below $1 \mathrm{kHz}[1,3]$. Similarly, the design of the feedback circuit can be adapted [8] in terms of architecture, ring resonator size and amount of rings used to provide the desired performance characteristics.

This work has been supported by the Deutsche Forschungsgemeinschaft (DFG, German Research Foundation) under Germany's Excellence Strategy via the Excellence Cluster 3D Matter Made to Order (EXC-2082/1 - 390761711), BMBF joint project PRIMA (13N14630), H2020 Photonic Packaging Pilot Line PIXAPP (731954) and by the European Research Council (ERC Consolidator Grant "TeraSHAPE” (773248).

\section{References}

[1] M. A. Tran, D. Huang, J. Guo, T. Komljenovic, P. A. Morton, and J. E. Bowers, "Ring-Resonator Based Widely-Tunable Narrow-Linewidth Si/InP Integrated Lasers," IEEE J. Sel. Top. Quantum Electron., vol. 26, no. 2, pp. 1-14, 2020.

[2] H. Guan et al., "Widely-tunable, Narrow-linewidth III-V/Silicon Hybrid External-cavity Laser for Coherent Communication," Opt. Express, vol. 26, no. 7, pp. 7920-7933, Apr. 2018.

[3] Y. Fan et al., "290 Hz Intrinsic Linewidth from an Integrated Optical Chip-based Widely Tunable InP-Si $\mathrm{N}_{4}$ Hybrid Laser," 2017 , p. JTh5C.9.

[4] N. Lindenmann et al., "Photonic Wire Bonding: A Novel Concept for Chip-scale Interconnects," Opt. Express, 2012.

[5] M. R. Billah et al., "Hybrid Integration of Silicon Photonics Circuits and InP Lasers by Photonic Wire Bonding," Optica, vol. 5, no. 7, pp. 876-883, Jul. 2018.

[6] M. Blaicher et al., "3D-Printed Ultra-Broadband Highly Efficient Out-of-Plane Coupler for Photonic Integrated Circuits," in Conference on Lasers and Electro-Optics, 2018, p. STh1A.1.

[7] K. Kikuchi, "Characterization of Semiconductor-laser Phase Noise and Estimation of Bit-error Rate Performance with Low-speed Offline Digital Coherent Receivers," Opt. Express, vol. 20, no. 5, pp. 5291-5302, Feb. 2012.

[8] T. Komljenovic et al., "Widely-Tunable Ring-Resonator Semiconductor Lasers," Appl. Sci., vol. 7, no. 7, 2017. 